\title{
Idiopathic Intracranial Hypertension and Obesity
}

\author{
Laura E. Andrews ${ }^{c}$ Grant T. Liu ${ }^{d, e}$ Melissa W. Ko ${ }^{\text {a-c }}$ \\ ${ }^{a}$ Department of Neurology, University Health Care Center, and bepartment of Ophthalmology, ' SUNY Upstate \\ Medical University, Syracuse, N.Y., and Departments of ${ }^{\mathrm{d}}$ Neurology and ${ }^{\mathrm{e}}$ Ophthalmology, Perelman School of \\ Medicine at the University of Pennsylvania, Philadelphia, Pa., USA
}

\begin{abstract}
Key Words
Idiopathic intracranial hypertension, pediatric .

Pseudotumor cerebri · Obesity · Weight gain · Recurrence
\end{abstract}

\begin{abstract}
Idiopathic intracranial hypertension $(\mathrm{IIH})$, also known as primary pseudotumor cerebri syndrome (PTCS), is a condition of unknown etiology which affects primarily overweight, reproductive-aged women and causes increased intracranial pressure (ICP). This review discusses the recently revised diagnostic criteria for PTCS for adults and children. Additionally, the role of obesity in the epidemiology, etiology, and management of IIH as well as the current knowledge of obesity profiles and markers in $\mathrm{IH}$ are reviewed. We also highlight the emerging, unifying theory of the neuroendocrine effects on the mineralocorticoid receptor to explain a possible mechanism for the increased cerebrospinal fluid production and ICP in secondary PTCS. @ 2014 S. Karger AG, Basel
\end{abstract}

\section{Introduction}

Idiopathic intracranial hypertension (IIH), also known as primary pseudotumor cerebri syndrome (PTCS) [1], is a disease of unknown etiology that causes increased intracranial pressure (ICP) without any identifiable cause. It tends to affect young, overweight women of reproductive

\section{KARGER}

E-Mail karger@karger.com

www.karger.com/hrp age, with symptoms of headache, nausea, vomiting, transient vision loss, impaired visual fields, photopsias, diplopia, and eye pain [2]. Other symptoms of IIH include pulse-synchronous tinnitus, and shoulder or arm pain [2, 3]. Ophthalmologic signs of IIH include decreased visual acuity, visual field loss, and papilledema. Permanent vision loss may affect up to $30 \%$ of patients [2], and sixth or seventh nerve palsies may also be present [2]. In the pediatric population, the signs and symptoms of IIH can be variable. Children can describe visual symptoms including transient visual loss, photophobia, and 'shimmering lights with colored centers' [4]. Headache is the main symptom among children with IIH and it has been documented in $62-91 \%$ of cases [5-7]. Of note, for unclear reasons there are reports of $\mathrm{IIH}$ without headache symptoms in children, either because the child is too young to articulate or because the headache is truly absent [8-11]. Children with IIH but without the headache tend to have more neurological signs, and vision loss at presentation is associated with a poorer prognosis [6]. Additionally, in pediatric cases of IIH the presentation may mimic a lesion in the posterior fossa with ataxia, facial palsy, nuchal rigidity, and torticollis [12].

This review encompasses the newly revised diagnostic criteria for PTCS in adults and children and the role of obesity in the epidemiology, etiology, and management of IIH. It also covers our current knowledge of obesity profiles and markers in IIH. Other recent reviews, including one on pediatric IIH [13] which we published in Hormone Re- (c) 2014 S. Karger AG, Basel

$1663-2818 / 14 / 0814-0217 \$ 39.50 / 0$
Melissa W. Ko, MD

Department of Neurology, University Health Care Center SUNY Upstate Medical University, 90 Presidential Plaza Syracuse, NY 13202 (USA)

E-Mail kom@upstate.edu 
Table 1. Updated 2013 diagnostic criteria for the PTCS [1]

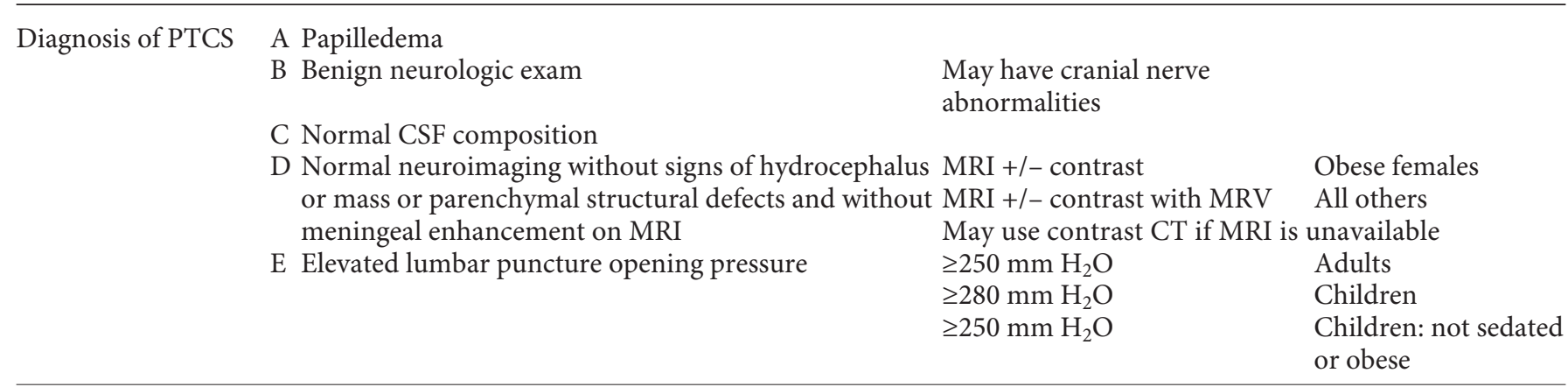

Diagnosis of PTCS 1 B to E from the above criteria are satisfied

without papilledema 2 Unilateral or bilateral abducens nerve palsy

Probable PTCS 1 A to D from the above criteria are satisfied

2 Normal lumbar puncture opening pressure

Suggested PTCS 1 B to E from the above criteria are satisfied

2 Neuroimaging shows at least 3 of the following:

Empty sella
Flattening of the posterior globe
Distension of the perioptic subarachnoid
space $+/-$ tortuous optic nerve
Transverse venous sinus stenosis

search in Paediatrics in 2010, can provide further scope regarding the multifactorial pathophysiology and treatment options of this disease in adults $[14,15]$ and in children.

\section{Search Strategy}

PubMed was utilized to search for IIH and pseudotumor cerebri each with: weight, obesity, pediatric obesity, weight gain, weight loss, gastric bypass surgery, weight loss surgery, aldosterone, and growth hormone. IIH was specifically searched with adiposity, leptin, cortisol, obstructive sleep apnea, economic cost, pathophysiology, diagnostic criteria, and epidemiology. Searches were performed both with a publication date filter of 1995 to the present and without a filter. The reference lists of identified articles were also searched for further relevant articles.

\section{Nomenclature and Diagnostic Criteria}

The diagnosis of PTCS was first characterized by Dandy [16] in 1937. The criteria were later modified by Smith [17] in 1985 (modified Dandy criteria), again by Fried- man and Jacobson [18] in 2002, and most recently by Friedman et al. [1] in 2013 (table 1). New terminology and criteria were necessary to incorporate advances in the knowledge of PTCS over the last decade, including insights of normal opening pressure in children, the characterization of typical neuroimaging abnormalities, and understanding of the features distinguishing intracranial hypertension with and without papilledema. Additionally, due to confusion with numerous terms utilized to describe the disorder, including 'pseudotumor cerebri,' 'idiopathic intracranial hypertension', and 'benign intracranial hypertension,' the authors of the new criteria felt the condition was best described using the umbrella term 'pseudotumor cerebri syndrome'. This term encompasses both primary (idiopathic) and secondary causes of intracranial hypertension (venous sinus thrombosis, medications, or medical conditions). Based on the new criteria, IIH is considered a subset within primary PTCS.

According to the newly proposed criteria, a 'definitive' diagnosis of PTCS requires: papilledema, a normal neurologic exam other than cranial nerve abnormalities, a normal cerebrospinal fluid (CSF) composition, an elevated lumbar puncture opening pressure $(\geq 280 \mathrm{~mm}$ CSF in children or $\geq 250 \mathrm{~mm}$ CSF in adults or unsedated, non- 
obese children), and normal neuroimaging. For the neuroimaging, there should be normal brain parenchyma (no evidence of hydrocephalus, mass or structural lesions, or meningeal enhancement) on MRI with and without gadolinum for typical patients (female and obese). For all other types of patients, MRI with and without gadolinium and MR venography should be performed. When MRI is unavailable or contraindicated, contrast-enhanced CT of the head may be used. PTCS is considered 'probable' if the above criteria are met in the presence of an opening pressure lower than specified for a 'definite' diagnosis. The diagnosis can also be made in the absence of papilledema if the patient has all of the above criteria including unilateral or bilateral abducens nerve palsy. In the absence of papilledema or sixth nerve palsy, the diagnosis of PTCS is 'suggested' if all other criteria are satisfied and the patient has at least $3 \mathrm{key}$ findings on neuroimaging (empty sella, flattening of the posterior aspect of the globe, distention of the perioptic subarachnoid space with or without a tortuous optic nerve, or transverse venous sinus stenosis) [1].

\section{PTCS Etiology}

While the etiology of IIH remains unknown, there have been many postulated theories. Proposed mechanisms that could explain the elevated ICP in IIH include increased cerebral volume (either interstitial fluid, blood or tissue), increased CSF volume due to increased production or resistance to CSF outflow, loss of cerebral autoregulation resulting in elevated cerebral arterial pressure, or increased cerebral venous pressure leading to increased CSF volume and decreased CSF outflow [19]. Secondary PTCS can be caused by many medical conditions and medication side effects, of which an exhaustive list is detailed in the literature $[2,18]$. Notably, disorders of the hypothalamic-pituitary-adrenal axis such as Addison's disease and Cushing's disease, and withdrawal from chronic use of corticosteroids, have been shown to be associated with the development of secondary PTCS [20]. Among medication associations, there are many case reports of secondary PTCS following the use of tetracycline compounds and hypervitaminosis A $[2,18]$.

A recent study proposed a theory unifying various neuroendocrine effects on the mineralocorticoid receptor (MR) to explain a possible mechanism for the increased CSF production and ICP in secondary PTCS [21]. The MR is abundant in choroid plexus (CP) epithelial cells, which regulate CSF production. Activation of the
MR or its downstream pathways can enhance and stimulate the generation of $\mathrm{Na} / \mathrm{K}$ ATPase pumps, which can lead to the movement of sodium ions at the CP apical membrane into the cerebral ventricle and actively create an osmotic gradient to drive CSF secretion and increase CSF pressure [21]. The authors hypothesize that this may be a key mechanism for secondary PTCS in patients with hyperaldosteronism, obesity, hypercortisolism, hypervitaminosis A, and recombinant growth hormone. For example, aldosterone, a mineralocorticoid (MC) responsible for $\mathrm{Na}^{+}$resorption and $\mathrm{K}^{+}, \mathrm{Ca}^{2+}$, and $\mathrm{Mg}^{2+}$ excretion at target tissues, including the epithelial cells of the $\mathrm{CP}$, can exert its biologic effects on these cells via the MR [21]. An increase in its activity in disorders of primary or secondary hyperaldosteronism may potentially directly affect the ICP in IIH. Support for this hypothesis includes numerous recent studies demonstrating that, in children and adults with primary or secondary hyperaldosteronism, those treated with spironolactone, an aldosterone receptor antagonist, had resolution of symptoms [22]. Additional pediatric cases further strengthen this link of IIH associated with hyperaldosteronism whereby children have been successfully controlled with spironolactone after failure of acetazolamide treatment. In one case the child had secondary hyperaldosteronism, and in the other case the child had metabolic syndrome, obesity, and hyperaldosteronism $[12,23]$.

The authors also postulate that various reported associations with IIH, including obesity, may all have a unified action on the MR pathway. CSF cortisol levels regulated by $11-\beta$-hydroxysteroid dehydrogenase, an abundant enzyme in the $\mathrm{CP}$ which converts inactive cortisone to cortisol, can act with high affinity at the MR in the CP. Thus, derangements of the corticosteroid axis through exogenous or endogenous mechanisms may lead to the development of IIH via this mechanism. Human fat, an active endocrine tissue, secretes MC-releasing factors, providing another possible link for elevated ICP in obese patients with IIH. Vitamin A has been shown to induce expression of neurosteroids in glial cell lines, which could also theoretically interact with MC receptors [21]. Finally, a relationship between IIH and recombinant growth hormone therapy has been reported numerous times and recombinant growth hormone has been regularly associated with increased plasma renin activity and aldosterone levels [21, 24-26]. Thus, this theory proposes that the above mentioned associations with IIH may all have a unified action on the MR pathway in the CP, resulting in altered CSF fluid dynamics and elevated ICP [21]. 
Table 2. Epidemiologic studies on IIH and obesity

\begin{tabular}{|c|c|c|c|c|}
\hline $\begin{array}{l}\text { Study location } \\
\text { Iowa, USA [27] }]^{2,4}\end{array}$ & \multicolumn{2}{|c|}{$\begin{array}{l}\text { IIH incidence in the general } \\
\text { population }\end{array}$} & \multicolumn{2}{|c|}{$\begin{array}{l}\text { IIH incidence in the overweight/obese population } \\
\text { or percent of IIH patients who are } \\
\text { overweight/obese }\end{array}$} \\
\hline Louisiana, USA [27] 3,4 & $1.07 / 100,000$ & & $14.85 / 100,000$ & Obese 9 \\
\hline Detroit Medical Center, USA [67] ${ }^{3,4}$ & - & & $90 \%$ & Obese \\
\hline Northern Ireland $[28]^{4}$ & $\begin{array}{l}0.5 / 100,000 \\
0.9 / 100,000\end{array}$ & q & - & \\
\hline Hokkaido, Japan (2 patients) $[31]^{1}$ & $\begin{array}{l}0.03 / 100,000 \\
(2 / 5,780,000)\end{array}$ & & - & \\
\hline Korea (10 patients) $[34]^{1,4}$ & - & & $\begin{array}{l}50 \% \\
7.1 \%\end{array}$ & $\begin{array}{l}\text { Overweight } \\
\text { Obese }\end{array}$ \\
\hline China (12 patients) $[35]^{1,4}$ & - & & $33 \%$ & Obese \\
\hline Libya $[32]^{2,4}$ & $\begin{array}{l}2.2 / 100,000 \\
4.3 / 100,000 \\
12 / 100,000\end{array}$ & $\begin{array}{l}\text { Q } \\
\text { RA } 9\end{array}$ & $21.4 / 100,000$ & Obese 9 \\
\hline
\end{tabular}

\section{Epidemiology and Obesity}

Many epidemiological studies have characterized the incidence of IIH and its association with obesity as defined by an elevated body mass index (BMI), although some studies have utilized other methods including the examiner's impression of the patient's weight (table 2). On average, the incidence of IIH in the general population is $0.5-1.0 / 100,000[8,27-30]$. The general popula- tion numbers appear to be lower in some Asian countries [31] and higher in Libya [32] and the UK [33]. Authors of both Italian [29] and UK [33] studies feel that the discrepancies between their IIH and obesity rates and published data could be due to variable obesity rates within their particular populations. The low incidence of IIH in Japan [31], and the lower prevalence of obesity among IIH patients in Korea [34] and China [35], is an interesting finding. Although these studies are quite small, there is some 
speculation that obesity does not play as prominent a role in the development of IIH in Asian patients. Kapoor [36], however, cautions that standard definitions of obesity based on the BMI may not necessarily be universally applicable to all races, and that obesity thresholds in Asian populations should perhaps be lower, thus increasing the number of Asian patients with IIH classified as overweight or obese. These trends within the Asian population may warrant further investigation.

Additionally, although there is less epidemiologic data on pediatric IIH, a recent German study found a similar incidence of $0.5 / 100,000$ [37]. The interest in pediatric $\mathrm{IIH}$ is increasing due to epidemic childhood obesity rates, with a rate of $16.9 \%$ in US children and adolescents in 2009-2010 [38]. Although prior evidence linking pediatric IIH to obesity has been inconclusive, a recent crosssectional study of 78 children and adolescents with IIH found that $73.1 \%$ were overweight or obese, with higher weight classes having progressively higher odds of disease [39]. White non-Hispanic race and female sex in patients older than 11 years were also risk factors. Given this evidence, authors speculate that childhood obesity rates may result in increased morbidity from IIH in this population [39].

Finally, with the striking tendency of IIH to afflict young obese women, a few studies have attempted to further characterize the condition by studying IIH in men. Kesler et al. [40] identified 18 men out of 134 patients with IIH and found that men and women had similar clinical features and outcomes, but far fewer men were overweight or obese ( $25 \%$ of males vs. $77.8 \%$ of females). Characterizations of weight, however, were made based on the examiner's impression, not the BMI. Another study also found that men with IIH had more severe vision loss and were less likely to be obese compared to women [41]. Bruce et al. [42], however, reported that in 66 men out of 721 patients with IIH, no difference in BMI was found between men and women, but men were more likely to have obstructive sleep apnea (OSA) (24\% of men vs. $4 \%$ of women) and twice as likely to suffer serious vision loss. Similarly, another study found that men with IIH were more likely to have OSA than age- and BMImatched controls, and they also have an increased prevalence of testosterone deficiency symptoms [43]. With conflicting results, it is unclear if obesity factors as greatly in the development of IIH in men compared to women [40-42]. There appears to be a consensus that men with IIH may be at a higher risk for severe vision loss and their management likely requires additional clinical surveillance for vision loss and OSA [41-43].

\section{Weight Gain and Weight Loss}

Regarding weight gain, Daniels et al. [44] found that, in 34 newly diagnosed IIH patients and 41 controls, IIH patients reported higher degrees of weight gain in the 12 months prior to the onset of IIH. Higher BMI and percentages of weight gain were also associated with a dose-dependent risk of developing IIH. Interestingly, both previously obese and nonobese patients $(\mathrm{BMI}<30)$ had a similar increased risk of developing IIH with only a small weight gain of 5-15\% [44]. Other studies have also reported an association between recent weight gain and the onset of IIH $[3,45,46]$. In a case-control study of 50 women with IIH, patients with a recurrence of IIH (26/50) had a higher $\mathrm{BMI}$ at recurrence compared to their BMI at diagnosis and at the initial resolution of symptoms [47]. On average, these patients had a $6 \%$ weight gain between resolution and recurrence, a finding which is similar to the numbers reported by Daniels [44] and actually corresponds with another study which found that just an average of $6 \%$ weight loss resulted in complete resolution of papilledema in IIH patients [48]. Conversely, patients without recurrence had a lower BMI at follow-up compared to diagnosis [47]. Of significance, in this study the average weight of patients without recurrence was greater than that of patients with recurrence throughout the study, suggesting that weight change may be equally important in the development and resolution of IIH compared to baseline weight [47]. Evidence supporting the importance of recent weight gain versus baseline weight also exists in the pediatric population. In a study of 14 pediatric patients with $\mathrm{IIH}$, all 5 patients with excess weight reported the majority of the weight gain within the 18 months prior to developing symptoms of IIH [49]. Despite these results, it is important to note that one third to one half of IIH patients do not exhibit any weight gain prior to the onset of their disease, although these data have often been based on self-reported weight $[28,29,44]$. The studies above suggest that both recent weight gain and baseline BMI are likely critical in IIH pathogenesis and underscore the importance of losing and maintaining weight loss.

Weight loss is also an important predictor of disease progression and visual outcomes. This was originally discussed in 1974 by Newborg [50], who utilized a rice reduction diet for the treatment of IIH. All patients on this low-calorie, low-salt diet had weight reduction and improvement of symptoms. More recently, Burdon et al. [51] followed 25 women with chronic, stable IIH through a 3-stage study, including 3 months each of: no intervention, a 425 -kcal diet, and a follow-up period. All women

Horm Res Paediatr 2014;81:217-225 
in the study who lost weight reduced their ICP and demonstrated improvement of papilledema, Pelli-Robson contrast, and Humphrey visual field mean deviations. The headache severity and frequency improved, analgesic use declined, and patients reported fewer symptoms of IIH. Importantly, only 4 of these women reduced their ICP to below $20 \mathrm{~cm} \mathrm{H}_{2} \mathrm{O}$, suggesting that patients may not need to reduce their ICP to normal levels to achieve dramatic improvement in the signs and symptoms of IIH [51]. In another retrospective study of 58 women with $\mathrm{IIH}$ who were followed for at least 6 months, patients were divided into 2 groups: those who had lost at least $2.5 \mathrm{~kg}$ in some 3-month interval during the study and those who had not [52]. Although there was no difference in final visual acuity outcomes between groups, the mean time to improvement of papilledema and visual fields was significantly lower in the weight loss group, again highlighting the importance of weight loss for the disease course. In another small study of 15 obese adult women with IIH treated with only weight loss and/or acetazolamide for 24 weeks, 11 of 15 patients had complete resolution or improvement of papilledema [48]. The amount of weight loss was associated with the degree of improvement of papilledema, and all patients with improvement of papilledema had unchanged normal or improved visual fields. As above [52], it was found that relatively small amounts of weight loss were of great benefit, as the average weight loss of those with complete resolution of papilledema was just $6.2 \%$ [48]. As a whole, the data supports the role of weight loss in the treatment of IIH. Although patients may require other treatment modalities, given the benefits of even modest weight loss on IIH disease progression and outcomes, this should be an area of emphasis for treating physicians.

\section{Degree of Obesity and Disease Outcomes}

There is still uncertainty as to whether the degree of obesity affects the severity of symptoms and disease outcomes, particular visual field defects. In a new study of 414 IIH patients, those with BMI $>40$ were more likely to present with severe papilledema and showed a trend of more severe visual loss at follow-up than less obese patients [53]. These authors also reported that for every 10 -unit increase in BMI the odds of severe vision loss increased by 1.4 times. In another recent study of 159 female patients with IIH [54], when comparing those with BMI $>40$ in the year before the diagnosis to those with $\mathrm{BMI}<40$, patients with $\mathrm{BMI}$ in the higher group had a higher prevalence of visual field defects. Another study of 34 patients also showed that severe obesity $(\mathrm{BMI}>40)$ was associated with worse visual outcomes [45]. Thus, it appears that those with morbid obesity $($ BMI $>40)$ have worse visual outcomes and this further underscores the importance of aggressively pursuing weight loss options in extremely obese patients.

\section{Bariatric Surgery and IIH}

With weight loss as the cornerstone in treating IIH, the role of weight loss surgery has become an important consideration. Sugerman et al. [55] has provided much of the data on the role of gastric surgery and resolution of IIH. In their 1995 study, 8 patients treated with acetazolamide underwent gastric bypass surgery. Preoperatively, 5 patients continued to have papilledema and all complained of headache with elevated CSF pressure. Following surgery, all patients had resolution of papilledema and lowering of the ICP [55]. These results demonstrated the possible utility of weight loss surgery in the treatment of IIH. Another study of 24 patients with severe obesity found that those who underwent bariatric surgery had a higher success rate for resolution of the symptoms of IIH compared to patients who had CSF peritoneal shunting [56]. Weight loss surgery also provides the additional benefit of resolution of other obesity-related comorbidities such as degenerative joint disease, hypertension, diabetes, and sleep apnea [56]. A review of 11 publications (62 patients) found that $92 \%$ of patients with IIH who underwent weight loss surgery had resolution of their symptoms [57]. Of the patients with complete data available, $97 \%$ had resolution of papilledema [57]. Additionally, although data on the use of bariatric surgery to successfully treat medically resistant $\mathrm{IIH}$ in pediatric patients is lacking, case reports do exist in adolescents. In one case, a 16-year-old obese female with IIH who failed both medical management and shunt placement had resolution of symptoms after bariatric surgery-induced weight loss [58]. Given the rising prevalence of extreme childhood obesity, further research on this treatment is needed. Altogether, the evidence supports the likely utility of weight loss surgery in treating IIH in obese patients, particularly when other strategies have failed.

\section{Adiposity and Obesity Markers}

Numerous studies have sought to characterize adiposity and markers of obesity in the setting of $\mathrm{IIH}$, including a comparison by Kesler et al. [59] of 44 female IIH pa- 
tients versus 383 obese female patients presenting to an obesity clinic or participating in a national health survey. As the BMI does not address regional fat distribution, this study compared waist-to-hip ratios and waist circumferences and found both were significantly lower in IIH patients versus obesity-matched controls. Pure lower-body adiposity was quite common in the IIH patients (45.4\%), but uncommon in the control obese participants (2.17.2\%) [59]. Another study also found significantly lower waist-to-hip ratios in IIH patients compared to obese controls despite the BMI of the IIH group being an average of 7 points higher [60]. Kesler et al. [59] contend that these results refute an often cited hypothesis of IIH pathogenesis that intra-abdominal fat increases intra-abdominal pressure, resulting in increased intracranial venous pressure $[15,61]$, since most IIH patients had a higher proportion of lower-body fat versus central obesity. Kesler et al. [59] also propose that the prevalence of an estrogenic gynecoid (pear-shaped) fat distribution and the propensity of IIH to affect obese women in their reproductive years support looking further into the role of estrogen in this disease. Peripheral conversion of androgens to estrogens in adipocytes, elevation of circulating estrogens in the reproductive years, the association of a gynecoid fat distribution with the onset of puberty [59], and reports of the onset of IIH in postmenopausal women following the initiation of hormone replacement therapy [62] lend support to the estrogen theory.

Obesity markers, particularly leptin, possibly contributing to IIH have been extensively examined. Lampl et al. [63] reported significantly higher serum leptin levels in IIH patients compared to obese controls. Ball et al. [60] also reported significantly higher CSF leptin levels in IIH patients compared to obese controls despite not being able to appreciate this finding among serum levels. Other groups also reported positive correlations between BMI and serum leptin levels, but their findings were either similar between IIH and control groups or failed to achieve statistical significance $[60,64,65]$. When Behbehani et al. [66] compared women with IIH to obese controls, there was no association between IIH and serum and CSF leptin levels following adjustments for age and BMI. The lack of multivariate regression analysis, small sample sizes, and variations in methodology in these studies make comparison of these results difficult. Additionally, no significant difference has been found between IIH and control obesity groups in serum insulin levels $[60,65]$ or ghrelin, a gastric hormone involved in weight regulation [65]. Further research in obesity markers may identify their significance in IIH.

\section{Conclusion}

While there has been great progress in our understanding of obesity and IIH since the description by Dandy [16] nearly 80 years ago, there is still much to be learned about the etiology, pathophysiology, and exact role of obesity in IIH, particularly within the pediatric population. The continued modification of the diagnostic criteria for PTCS reflects this progress and will likely help physicians make more a more accurate diagnosis. In the last decade alone, we have learned that there may be a lesser role of obesity in Asians [31,34-36] and men [40,41], but men and the morbidly obese may experience more severe vision loss [41, 42, 45, 53, 54]. We continue to see a strong association between IIH and recent weight gain $[3,44,45]$ (even modest gains of 6\%) [47], but encouragingly the converse appears to be true, as a similar amount of weight loss results in significant improvement [48]. For the morbidly obese IIH patient, bariatric surgery is a likely effective treatment [55-57] when noninvasive strategies have failed. Currently, studies of obesity markers in IIH patients have been inconclusive; however, neuroendocrine interactions on CSF secretion via MR activation is an area of active interest. Looking ahead, publication of the highly anticipated results of the Idiopathic Intracranial Hypertension Treatment Trial (IIHTT), a multicenter, prospective, randomized, placebo-controlled trial will likely further our understanding of IIH and its effective treatment strategies.

References

1 Friedman DI, Liu GT, Digre KB: Revised diagnostic criteria for the pseudotumor cerebri syndrome in adults and children. Neurology 2013;81:1-7.

2 Degnan AJ, Levy LM: Pseudotumor cerebri: brief review of clinical syndrome and imaging findings. AJNR Am J Neuroradiol 2011;32: 1986-1993.

3 Giuseffi V, Wall M, Siegel PZ, Rojas PB: Symptoms and disease associations in idiopathic intracranial hypertension (pseudotumor cerebri): a case-control study. Neurology 1991;41:239-244.

-4 Soler D, Cox T, Bullock P, Calver DM, Robinson RO: Diagnosis and management of benign intracranial hypertension. Arch Dis Child 1998;78:89-94.

5 Babikian P, Corbett J, Bell W: Idiopathic intracranial hypertension in children: the Iowa experience. J Child Neurol 1994;9:144149.

-6 Lim M, Kurian M, Penn A, Calver D, Lin JP: Visual failure without headache in idiopathic intracranial hypertension. Arch Dis Child 2005;90:206-210. 
7 Phillips PH, Repka MX, Lambert SR: Pseudotumor cerebri in children. J AAPOS 1998;2: 33-38.

-8 Radhakrishnan K, Ahlskog JE, Cross SA, Kurland LT, O'Fallon WM: Idiopathic intracranial hypertension (pseudotumor cerebri): descriptive epidemiology in Rochester, Minn, 1976 to 1990. Arch Neurol 1993;50:78-80.

$\checkmark$ Lessell S: Pediatric pseudotumor cerebri (idiopathic intracranial hypertension). Surv Ophthalmol 1992;37:155-166.

10 Kesler A, Fattal-Valevski A: Idiopathic intracranial hypertension in the pediatric population. J Child Neurol 2002;17:745-748.

11 Salman MS, Kirkham FJ, MacGregor DL: Idiopathic 'benign' intracranial hypertension: case series and review. J Child Neurol 2001; 16:465-470.

12 Salpietro V, Mankad K, Kinali M, et al: Pediatric idiopathic intracranial hypertension and the underlying endocrine-metabolic dysfunction: a pilot study. J Pediatr Endocrinol Metab 2014;27:107-115.

$\checkmark 13$ Ko MW, Liu GT: Pediatric idiopathic intracranial hypertension (pseudotumor cerebri). Horm Res Paediatr 2010;74:381-389.

-14 Biousse V, Bruce BB, Newman NJ: Update on the pathophysiology and management of idiopathic intracranial hypertension. J Neurol Neurosurg Psychiatry 2012;83:488-494.

$\checkmark 15$ Ooi LY, Walker BR, Bodkin PA, Whittle IR: Idiopathic intracranial hypertension: can studies of obesity provide the key to understanding pathogenesis? Br J Neurosurg 2008; 22:187-194.

16 Dandy WE: Intracranial pressure without brain tumor: diagnosis and treatment. Ann Surg 1937;106:492-513.

$\checkmark 17$ Smith JL: Whence pseudotumor cerebri? J Clin Neuroophthalmol 1985;5:55-56.

18 Friedman DI, Jacobson DM: Diagnostic criteria for idiopathic intracranial hypertension. Neurology 2002;59:1492-1495.

19 Walker RW: Idiopathic intracranial hypertension: any light on the mechanism of the raised pressure? J Neurol Neurosurg Psychiatry 2001;71:1-5.

-20 Zada G, Tirosh A, Kaiser UB, Laws ER, Woodmansee WW: Cushing's disease and idiopathic intracranial hypertension: case report and review of underlying pathophysiological mechanisms. J Clin Endocrinol Metab 2010;95:4850-4854.

-21 Salpietro V, Polizzi A, Berte LF, et al: Idiopathic intracranial hypertension: a unifying neuroendocrine hypothesis through the adrenal-brain axis. Neuro Endocrinol Lett 2012; 33:569-573.

22 Khan MU, Khalid H, Salpietro V, Weber KT: Idiopathic intracranial hypertension associated with either primary or secondary aldosteronism. Am J Med Sci 2013;346:194-198.

$\checkmark 23$ Salpietro V, Ruggieri M, Sancetta F, et al: New insights on the relationship between pseudotumor cerebri and secondary hyperaldosteronism in children. J Hypertens 2012;30:629630.
24 Malozowski S, Tanner LA, Wysowski D, Fleming GA: Growth hormone, insulin-like growth factor I, and benign intracranial hypertension. N Engl J Med 1993;329:665-666.

25 Darendeliler F, Karagiannis G, Wilton P: Headache, idiopathic intracranial hypertension and slipped capital femoral epiphysis during growth hormone treatment: a safety update from the KIGS database. Horm Res 2007;68(suppl 5):41-47.

26 Besch D, Makowski C, Steinborn MM, Bonfig W, Sadowski B: Visual loss without headache in children with pseudotumor cerebri and growth hormone treatment. Neuropediatrics 2013;44:203-207.

27 Durcan FJ, Corbett JJ, Wall M: The incidence of pseudotumor cerebri: population studies in Iowa and Louisiana. Arch Neurol 1988;45: 875-877.

28 Craig JJ, Mulholland DA, Gibson JM: Idiopathic intracranial hypertension: incidence, presenting features and outcome in Northern Ireland (1991-1995). Ulster Med J 2001;70: 31-35.

29 Carta A, Bertuzzi F, Cologno D, Giorgi C, Montanari E, Tedesco S: Idiopathic intracranial hypertension (pseudotumor cerebri): descriptive epidemiology, clinical features, and visual outcome in Parma, Italy, 1990 to 1999. Eur J Ophthalmol 2004;14:48-54.

30 Kesler A, Gadoth N: Epidemiology of idiopathic intracranial hypertension in Israel. J Neuroophthalmol 2001;21:12-14.

- 31 Yabe I, Moriwaka F, Notoya A, Ohtaki M, Tashiro K: Incidence of idiopathic intracranial hypertension in Hokkaido, the northernmost island of Japan. J Neurol 2000;247:474475.

32 Radhakrishnan K, Thacker AK, Bohlaga NH, Maloo JC, Gerryo SE: Epidemiology of idiopathic intracranial hypertension: a prospective and case-control study. J Neurol Sci 1993; 116:18-28.

33 Raoof N, Sharrack B, Pepper IM, Hickman SJ: The incidence and prevalence of idiopathic intracranial hypertension in Sheffield, UK. Eur J Neurol 2011;18:1266-1268.

34 Kim TW, Choung HK, Khwarg SI, Hwang JM, Yang HJ: Obesity may not be a risk factor for idiopathic intracranial hypertension in Asians. Eur J Neurol 2008; 15:876-879.

- 35 Liu IH, Wang AG, Yen MY: Idiopathic intracranial hypertension: clinical features in Chinese patients. Jpn J Ophthalmol 2011;55: 138-142.

36 Kapoor KG: Regarding 'obesity may not be a risk factor for idiopathic intracranial hypertension in Asians'. Eur J Neurol 2009;16:e1, author reply e2.

37 Tibussek D, Distelmaier F, von Kries R, Mayatepek E: Pseudotumor cerebri in childhood and adolescence - results of a Germany-wide ESPED-survey. Klin Padiatr 2013;225:81-85. 38 Ogden CL, Carroll MD, Kit BK, Flegal KM: Prevalence of obesity and trends in body mass index among US children and adolescents, 1999-2010. JAMA 2012;307:483-490.
39 Brara SM, Koebnick C, Porter AH, LangerGould A: Pediatric idiopathic intracranial hypertension and extreme childhood obesity. J Pediatr 2012;161:602-607.

40 Kesler A, Goldhammer Y, Gadoth N: Do men with pseudomotor cerebri share the same characteristics as women? A retrospective review of 141 cases. J Neuroophthalmol 2001; 21:15-17.

41 Digre KB, Corbett JJ: Pseudotumor cerebri in men. Arch Neurol 1988;45:866-872.

42 Bruce BB, Kedar S, Van Stavern GP, et al: Idiopathic intracranial hypertension in men. Neurology 2009;72:304-309.

43 Fraser JA, Bruce BB, Rucker J, et al: Risk factors for idiopathic intracranial hypertension in men: a case-control study. J Neurol Sci 2010;290(1-2):86-89.

44 Daniels AB, Liu GT, Volpe NJ, et al: Profiles of obesity, weight gain, and quality of life in idiopathic intracranial hypertension (pseudotumor cerebri). Am J Ophthalmol 2007; 143:635-641.

45 Rowe FJ, Sarkies NJ: The relationship between obesity and idiopathic intracranial hypertension. Int J Obes Relat Metab Disord 1999;23:54-59.

46 Ireland B, Corbett JJ, Wallace RB: The search for causes of idiopathic intracranial hypertension: a preliminary case-control study. Arch Neurol 1990;47:315-320.

47 Ko MW, Chang SC, Ridha MA, et al: Weight gain and recurrence in idiopathic intracranial hypertension: a case-control study. Neurology 2011;76:1564-1567.

48 Johnson LN, Krohel GB, Madsen RW, March GA Jr: The role of weight loss and acetazolamide in the treatment of idiopathic intracranial hypertension (pseudotumor cerebri). Ophthalmology 1998;105:23132317.

49 Salpietro V, Chimenz R, Arrigo T, Ruggieri M: Pediatric idiopathic intracranial hypertension and extreme childhood obesity: a role for weight gain. J Pediatr 2013;162:1084.

50 Newborg B: Pseudotumor cerebri treated by rice reduction diet. Arch Intern Med 1974; 133:802-807.

51 Sinclair AJ, Burdon MA, Nightingale PG, et al: Low energy diet and intracranial pressure in women with idiopathic intracranial hypertension: prospective cohort study. BMJ 2010; 341:c2701.

52 Kupersmith MJ, Gamell L, Turbin R, Peck V, Spiegel P, Wall M: Effects of weight loss on the course of idiopathic intracranial hypertension in women. Neurology 1998;50:10941098.

53 Szewka AJ, Bruce BB, Newman NJ, Biousse V: Idiopathic intracranial hypertension: relation between obesity and visual outcomes. J Neuroophthalmol 2013;33:4-8.

54 Baldwin MK, Lobb B, Tanne E, Egan R: Weight and visual field deficits in women with idiopathic intracranial hypertension. J Womens Health (Larchmt) 2010;19:18931898. 
55 Sugerman HJ, Felton WL 3rd, Salvant JB Jr, Sismanis A, Kellum JM: Effects of surgically induced weight loss on idiopathic intracranial hypertension in morbid obesity. Neurology 1995;45:1655-1659.

56 Sugerman HJ, Felton WL 3rd, Sismanis A, Kellum JM, DeMaria EJ, Sugerman EL: Gastric surgery for pseudotumor cerebri associated with severe obesity. Ann Surg 1999;229: 634-640, discussion 640-642.

57 Fridley J, Foroozan R, Sherman V, Brandt ML, Yoshor D: Bariatric surgery for the treatment of idiopathic intracranial hypertension. J Neurosurg 2011;114:34-39.

58 Chandra V, Dutta S, Albanese CT, Shepard E Farrales-Nguyen S, Morton J: Clinical resolution of severely symptomatic pseudotumor cerebri after gastric bypass in an adolescent. Surg Obes Relat Dis 2007;3:198-200.

59 Kesler A, Kliper E, Shenkerman G, Stern N: Idiopathic intracranial hypertension is associated with lower body adiposity. Ophthalmology 2010;117:169-174.
60 Ball AK, Sinclair AJ, Curnow SJ, et al: Elevated cerebrospinal fluid (CSF) leptin in idiopathic intracranial hypertension (IIH): evidence for hypothalamic leptin resistance? Clin Endocrinol (Oxf) 2009;70:863-869.

61 Sugerman HJ, DeMaria EJ, Felton WL 3rd, Nakatsuka M, Sismanis A: Increased intraabdominal pressure and cardiac filling pressures in obesity-associated pseudotumor cerebri. Neurology 1997;49:507-511.

62 Sheehan JP: Hormone replacement treatment and benign intracranial hypertension. Br Med J (Clin Res Ed) 1982;284:1675-1676.

63 Lampl Y, Eshel Y, Kessler A, et al: Serum leptin level in women with idiopathic intracranial hypertension. J Neurol Neurosurg Psychiatry 2002;72:642-643.
64 Dhungana S, Sharrack B, Woodroofe N: Cytokines and chemokines in idiopathic intracranial hypertension. Headache 2009;49:282285.

65 Subramanian PS, Goldenberg-Cohen N, Shukla S, Cheskin LJ, Miller NR: Plasma ghrelin levels are normal in obese patients with idiopathic intracranial hypertension (pseudotumor cerebri). Am J Ophthalmol 2004;138: 109-113.

66 Behbehani R, Mabrook A, Abbas JM, AlRammah T, Mojiminiyi O, Doi SA: Is cerebrospinal fluid leptin altered in idiopathic intracranial hypertension? Clin Endocrinol (Oxf) 2010;72:851-852.

67 Galvin JA, Van Stavern GP: Clinical characterization of idiopathic intracranial hypertension at the Detroit Medical Center. J Neurol Sci 2004;223:157-160.

-68 Celebisoy N, Secil Y, Akyurekli O: Pseudotumor cerebri: etiological factors, presenting features and prognosis in the western part of Turkey. Acta Neurol Scand 2002;106:367-370. 